\title{
Comparison of Serum Biochemical and Haematological Analyses in Patients with Age-Related Macular Degeneration and Diabetic Retinopathy
}

\author{
Muberra Akdogan ${ }^{1}$, Yasemin Ustundag ${ }^{2}$, M. Cem Sabaner ${ }^{1}, \&$ Mustafa Dogan ${ }^{1}$ \\ 1 Department of Opthhalmology, Afyonkarahisar Health Science University, Faculty of Medicine, \\ Afyonkarahisar, Turkey \\ ${ }^{2}$ Department of Clinical Biochemistry, University of Health Sciences, Bursa Yuksek Ihtisas Training and \\ Research Hospital, Bursa, Turkey \\ Correspondence: Muberra Akdogan, Department of Opthhalmology, Afyonkarahisar Health Science University, \\ Faculty of Medicine, Zafer Kulliyesi, Dortyol M. 2078. Sok Afyonkarahisar 03320, Turkey. Tel: \\ 0090272-246-3301. Fax: 0090272-228-1429. E-mail: mbrakdogan@yahoo.com; makdogan@aku.edu.tr
}

Received: April 4, 2019

Accepted: April 29, 2019

Online Published: May 11, 2019

doi:10.5539/jmbr.v9n1p41

URL: https://doi.org/10.5539/jmbr.v9n1p41

\begin{abstract}
We evaluated biochemical analysis results with the aim of discovering serum levels that have possible effects and differences on age-related macular degeneration (AMD) and diabetic retinopathy (DRP).

A retrospective case-control study was conducted between January 2017 and January 2018 on a total of 114 patients (84 DRP, 30 AMD) and 24 age and sex-matched control individuals. Four groups were created; 52 patients with proliferative DRP (PDR), 32 patients with nonproliferative PDR (non-PDR), 30 patients with wet AMD and 24 control individuals. Serum biochemical (HbA1C, fasting glucose, AST, ALT, C-reactive protein, albumin, total protein, uric acid, triglyceride, HDL, LDL, total cholesterol, Na, K, urine albumin) and complete blood count (CBC) analyses were performed at the time of diagnosis. Descriptive statistics, one-way ANOVA and Kruskal-Wallis tests were performed using SPSS to analyse data (Version 22.0).
\end{abstract}

The mean age of patients was 63.3 years \pm 6.4 (49-91year), and that of control individuals was 65.3 years \pm 9.3 (50-88 year). Post hoc analysis showed statistically significant differences in $\mathrm{HbAlc}$ and fasting glucose levels among PDR-AMD, PDR-control, non-PDR-AMD, and non-PDR-control groups (whole, $P<0.001$ ). Statistically significant differences in $\mathrm{RBC}, \mathrm{Hb}$, and Htc between PDR-control $(P=0.005, P=0.027 ; P=0.001)$, and non-PDR-control groups were also found $(P=0.005 ; P=0.003, P=0.045)$, respectively.

Differences among values may be attributed to the different pathophysiologies of the DRP and AMD diseases, as has been established for serum values. An intact versus a defective blood retinal barrier is thought to be implicated in these results.

Keywords: Diabetic Retinopathy, Age-Related Macular Degeneration, Uric Acid

\section{Introduction}

Diabetes is a life-threatening chronic disease with microvascular (retinopathy, nephropathy and neuropathy) and macrovascular complications leading to increased risk of cardiovascular disease, and an estimated 592 million people worldwide will have diabetes mellitus (DM) by 2035 (Guariguata et al., 2014). Diabetic retinopathy (DRP) is the most common microvascular complication of diabetes mellitus. Prolonged exposure to diabetes-related glucose and lipid metabolism changes results in excess free glycation products and free radicals that may lead to capillary changes and damage to the endothelium of capillaries, thickening of basal membranes and increased platelet aggregation. These, in turn, can lead to retinal ischemia secondary to vaso occlusion and DRP by damaging the microvascular structure of the retina (Sivaprasad et al., 2012; Stewart, 2010). DRP is the leading cause of preventable blindness in all age groups, and a significant burden on health care (Stewart, 2010). If diabetic patients were to receive follow-ups by an ophthalmologist at proper intervals, visual loss could be prevented via prophylactic diagnostic and therapeutic tools. Diabetes mellitus can be kept under control with strict diet control and lifestyle changes; in this manner, DRP development can thereby be prevented (Asif, 2014). 
Age-related macular degeneration (AMD) is one of the leading causes of progressive, bilateral blindness in elderly people (Mitchell et al., 2018). There are different classifications of the disease. The Age Related Eye Diseases Study (AREDS) simplified severity scale was published in 2005 and is based on data from 3212 participants of the AREDS clinical trial population (Liew et al., 2016). Meanwhile, Klein et al. (2014), describe methods to the classification of AMD phenotypes to reduce method -induced heterogeneity among studies, and divided AMD into 4 groups according to the criteria. Dry AMD is characterized primarily by increased drusen formation on externalretinal cells. Late stage dry-AMD consists of geographic atrophy (GA) characterized by external retinal and choroidal atrophy, especially in the photoreceptor layer, RPE and choriocapillaris. Exudative (wet) AMD is characterized by choroidal neovascularization, RPE detachment and submacular disciform scarring (Liew et al., 2016; Klein et al., 2014).

The retina is highly sensitive to oxidative stress due to its high oxygen consumption and constant exposure to light. Aging is associated with biological changes in the eye, including cumulative oxidative stress (Amanita et al., 2016). Meanwhile, enzymatic antioxidants and nonenzymatic antioxidants such as albumin and uric acid help defend the body against oxidative stress.

Normal aging increases the thickness of the Bruch's membrane through the accumulation of normal or abnormal extracellular matrix (ECM) material, a lipid-rich substance resulting from oxidative and nonenzymatic glycosylation. Lipid accumulation in retinal pigment epithelium (RPE) cells combined with oxidative stress act synergistically in the pathogenesis of AMD (Amanita et al., 2016; Kunchithapautham et al., 2014; Ambati et al., 2012).

Elevations in levels of local and systemic biomarkers indicate that oxidative stress and chronic inflammation is involved in the pathogenesis of AMD (Mitchell et al., 2018; Kunchithapautham et al., 2014; Ambati et al., 2012; Čolak et al., 2017).

To date, there are cross-sectional studies reporting that there may be a relationship between the variability in serum biochemical parameters, serum lipoproteins, inflammatory markers, $\mathrm{CBC}$ parameters and the risk of DR and AMD (Colijn et al., 2019; Paun et al., 2015; Boekhoorn et al., 2017; Prakash et al., 2017; Traveset et al., 2016). However, epidemiological data have been inconsistent regarding the associations of CRP, CBC parameters, serum lipid levels, DR and AMD (Colijn et al., 2019; Paun et al., 2015; Boekhoorn et al., 2007; Prakash et al., 2017; Traveset, et al., 2016).

We evaluated biochemical analysis results with the aim of discovering serum levels that have possible effects and differences on AMD and DRP.

\section{Material and Methods}

A retrospective case-control study was conducted at the Bursa Yuksek Ihtisas Training and Research Hospital, department of ophthalmology between January 2017 and February 2019 on a total of 138 patients PDR, non-PDR and wet AMD with mean age of $65 \pm 10$ years and 24 healthy individuals sex and age-matched control group with mean age $65 \pm 9.3$ years. The study group consisted of patients who are screened in the department of retina for diabetic retinopathy and AMD.

The Yuksek Ihtisas Training and Research Hospital Ethics Committee, Bursa, Turkey approved this study, we followed tenets of the Declaration of Helsinki (Ref: 2011/KAEK/25 dated 03/03/2019). A diagnosis of type 2 diabetes was made according to the 2016 standards provided by the American Diabetes Association. Categories of AMD was made according to the AREDS study on the exam findings of drusen, atrophy, and neovascularization (a-no AMD fewer than 5 small drusen $<65 \mu \mathrm{m}$, b-mild AMD multipl small drusen 63-124 $\mu \mathrm{m}$, c-intermediate AMD extensive intermediate sized drusen more than $>125 \mu \mathrm{m}$, d-advanced AMD with two categories central geographic atrophy konown as advanced dry and choroideal neovascularization known as wet or advanced-exudative form of AMD. Patients with AMD were included those with advanced-exudative AMD subgroup. Patients with dry type were not included in this study. All patients received a full ophthalmologic examination that included visual acuity, slit-lamp examination and indirect fundoscopic examination. A diagnosis of PDR, non-PDR or AMD was reached according to fundus fluorescein angiography (FFA) and SD-OCT (Heidelberg Engineering OCT 2 Module $85.000 \mathrm{~Hz}$ ) findings after detailed direct and indirect fundus examinations on dilated pupillae (Ambati et al., 2012; Čolak et al., 2017; Colijn et al., 2019; Paun et al., 2015; Boekhoorn et al., 2007; Prakash et al., 2017; Traveset et al., 2016; Magri et al., 2012). The control group were recruited from employees of the our hospital, their relatives and who were without any signs of AMD, DRP our ophthalmology department out of patients.

Inclusion Criteria: Patients with spherical refraction between -3.0 and +3.0 dioptres, an axial length (AL) between 23 and $25 \mathrm{~mm}$, and normal intraocular pressure $(12-22 \mathrm{mmHg}$ ) with a diagnosis of advanced-exudative 
form AMD, non-PDR or PDR between 45 and 88 years of age were included.

Exclusion Criteria: Type 1 diabetic patients, those with organ failure, liver disease, stroke, a any of ocular disease (retinal artery or vein occlusion, glaucoma, etc.) other than AMD and DRP, presence of systemic disease other than DM, a history of ocular surgery, alcohol intake or smoking, or a history of taking non-diabetic medications (including vitamin supplements for AMD) within the last 3 months.

Sample Size Calculation: The sample size was calculated according to es $=0.25, .9=0.05, \mathrm{p}=0.65$ (G Power 3.1.9.2).

Participants were divided into four groups as follows: 52 patients with PDR (group 1), 32 patients with non-PDR (group 2), 30 patients with wet AMD (group 3) and 24 age- and sex-matched control individuals (group 4).

Demographic data were obtained from patients or their medical records. BMI was calculated as weight in kilograms divided by height in meters squared. Results from serum biochemical and complete blood count analyses, performed at the time of diagnosis in the retina clinic, were evaluated.

\section{Sample Collection}

Whole blood samples of at least $2 \mathrm{cc}$ from 138 patients were collected with an injector for routine systemic biochemical analysis. Serum separator tubes were used to prevent haemolysis (SSTs; BD, Vacutainer SST, reference number: 367986; BD Biosciences); blood was added by removing the cap and transferring the sample to the tube with the direct injector with the needle tip removed. Additional samples were taken in two K3-EDTA $4 \mathrm{~mL}$ tubes (Vacuette, Greiner Bio-One, Austria) for HbAlc and CBC measurements. SSTs were centrifuged after coagulation upon standing for at least 10 minutes. All SSTs and one K-3EDTA tube were centrifuged at 4000rpm for 10 minutes by centrifuge device (Nüve, NF 1200 R, Ankara, Turkey). The other K3-EDTA tube was set aside for CBC analysis and not centrifuged. All SST and K3-EDTA tubes were delivered to the biochemistry laboratory without shaking. A complete urine sample was also collected and sent to the biochemistry laboratory for urine microalbumin analysis.

\section{Biochemical and Haematological Study and Analysis}

Whole biochemical serum analyses were all performed using the same device (Architect C16000, Abbott Diagnostics, Abbott Park, IL, USA). Levels of glucose (mg/dL), blood urine nitrogen (BUN) (mg/dL), creatinine $(\mathrm{mg} / \mathrm{dL})$, alanine aminotransferase (ALT) $(\mathrm{U} / \mathrm{L})$, aspartate aminotransferase (AST) $(\mathrm{U} / \mathrm{L})$, albumin $(\mathrm{mg} / \mathrm{dL})$, total protein $(\mathrm{mg} / \mathrm{dL}), \mathrm{CRP}(\mathrm{mg} / \mathrm{L})$, uric acid $(\mathrm{mg} / \mathrm{dL})$, sodium $(\mathrm{Na})(\mathrm{mEq} / \mathrm{L})$, potassium $(\mathrm{K})(\mathrm{mEq} / \mathrm{L})$, total cholesterol $(\mathrm{mg} / \mathrm{dL}), \mathrm{HDL}$ (high density lipoprotein) (mg/dL), LDL (low density lipoprotein) (mg/dL), triglyceride (mg/dL), and urine microalbumin $(\mathrm{mg} / \mathrm{L}$ ) were determined using commercially available assay kits (Abbott Diagnostics, Abbott Park, IL, USA) with an Architect C16000 autoanalyzer (Abbott Diagnostics, Abbott Park, IL, USA).

HbAlc measurements were performed by using the HPLC technique in an Adams HA-8160 analyser (Arkray KDK, Shiga, Japan).

CBC analysis was performed by a Coulter LH 780 Haematology Analyzer (Beckman Coulter Ireland Inc. Mervue, Galway, Ireland).

\section{Statistical Methods}

Data were recorded and analysed using the statistical package program (SPSS) version 22.0 (SPSS, Chicago, USA). Descriptive statistical methods (mean, standard deviation) were used to evaluate the data. Distribution of the data was examined by the Shapiro-Wilk test and found to be abnormally distributed. A parametric one-way ANOVA test was used to determine distribution parameters for comparison of whole groups. A non-parametric Kruskal-Wallis test was used for comparison of whole groups due to abnormal distribution parameters. Intergroup analyses were performed using a Bonferroni post hoc test for normal distribution, and a Dunn's test for abnormally distributed data. Pearson's or Spearman's correlation coefficients were calculated to evaluate the relationship between variables. The evaluations were made at the $95 \%$ confidence interval, and a $P$-value of less than 0.05 was considered statistically significant.

\section{Results}

The mean age of patients was 63.3 years \pm 6.4 (groups 1,2 and 3 ), and that of healthy individuals was 65.3 years \pm 9.3. The mean age of patients in group 1 was 62.6 years \pm 7.5 , in group 2 was 60.9 years \pm 6.2 , in group 3 was 66.8 years \pm 5.4 , and in group 4 was 65.3 years $\pm 9.3(P=0.076)$.

BMI $\left(\mathrm{kg} / \mathrm{m}^{2}\right)$ parameter was $30.3 \pm 5.3$ in group 1, 30.0 \pm 5.1 in group 2, $28.6 \pm 4.7$ in group 3 and $30.0 \pm 5.5$ in group $4(P=0.449)$. The distribution of demographic data and test results are shown in Table 1 . 
Table 1. Demographics of study sample, and results of biochemical analysis

\begin{tabular}{|c|c|c|c|c|c|}
\hline Characteristics & $\operatorname{PDR}(n=52)$ & non-PDR $(n=32)$ & $\operatorname{AMD}(n=30)$ & Control individuals $(n=24)$ & $P *$ value \\
\hline Age \pm Mean (SD) & $62.6 \pm 7.5$ & $60.9 \pm 6.2$ & $66.8 \pm 5.4$ & $65.3 \pm 9.3$ & 0.076 \\
\hline Male/female & $21 / 31$ & $12 / 20$ & $12 / 18$ & $10 / 14$ & \\
\hline Duration of Diabetes & $15 \pm 5$ & $13 \pm 4$ & - & - & 0.057 \\
\hline BMI $\left(\mathrm{kg} / \mathrm{m}^{2}\right)$ & $30.3 \pm 5.3$ & $30.0 \pm 5.1$ & $28.6 \pm 4.7$ & $30.0 \pm 5.5$ & 0.449 \\
\hline Hba1c (\%) & $8.4 \pm 1.9$ & $8.1 \pm 1.9$ & $5.6 \pm 0.5$ & $5.7 \pm 0.5$ & $P<0.001$ \\
\hline Fasting Glucose (mg/dl) & $201 \pm 87$ & $179 \pm 71$ & $106 \pm 70$ & $98 \pm 33$ & $P<0.001$ \\
\hline AST (U/L) & $20 \pm 6$ & $22 \pm 8$ & $21 \pm 9$ & $19 \pm 5$ & 0.253 \\
\hline $\mathbf{A L T}^{+}(\mathrm{U} / \mathrm{L})$ & $20 \pm 10$ & $21 \pm 11$ & $20 \pm 16$ & $18 \pm 6$ & ${ }^{+} 0.096$ \\
\hline $\mathbf{C R P}^{+}(\mathrm{mg} / \mathrm{L})$ & $6.3 \pm 3.4$ & $5.7 \pm 3.2$ & $4.2 \pm 3.0$ & $5.8 \pm 4.2$ & ${ }^{+} 0.110$ \\
\hline BUN (mg/dl) & $18.1 \pm 7.9$ & $15.6 \pm 7.6$ & $17.5 \pm 16.8$ & $17.7 \pm 9.3$ & 0.841 \\
\hline Creatinine (mg/dl) & $1.0 \pm 0.4$ & $0.9 \pm 0.2$ & $0.9 \pm 0.2$ & $0.8 \pm 0.2$ & 0.006 \\
\hline Albumin (mg/dl) & $4.1 \pm 0.3$ & $4.2 \pm 0.3$ & $4.4 \pm 0.3$ & $4.3 \pm 0.2$ & 0.004 \\
\hline Total protein (mg/dl) & $7.1 \pm 0.5$ & $7.1 \pm 0.4$ & $7.3 \pm 0.6$ & $7.2 \pm 0.2$ & 0.284 \\
\hline Uric acid (mg/dl) & $5.9 \pm 1.8$ & $5.2 \pm 1.5$ & $5.3 \pm 1.4$ & $5.2 \pm 1.6$ & 0.127 \\
\hline Triglyceride $^{+}(\mathrm{mg} / \mathrm{dl})$ & $192 \pm 107$ & $169 \pm 74$ & $140 \pm 52$ & $147 \pm 57$ & ${ }^{+} 0.190$ \\
\hline HDL (mg/dl) & $49.4 \pm 12.1$ & $46.7 \pm 10.8$ & $48.7 \pm 13.2$ & $49.1 \pm 11.2$ & 0.799 \\
\hline $\mathbf{L D L}(\mathrm{mg} / \mathrm{dl})$ & $119 \pm 47$ & $145 \pm 51$ & $121 \pm 52$ & $137 \pm 32$ & 0.088 \\
\hline Total cholesterol (mg/dl) & $204 \pm 47$ & $224 \pm 66$ & $184 \pm 43$ & $200 \pm 30$ & 0.062 \\
\hline $\mathbf{N a}(\mathrm{mEq} / \mathrm{L})$ & $139 \pm 3.9$ & $139 \pm 2.7$ & $140 \pm 1.7$ & $140 \pm 2.7$ & 0.101 \\
\hline $\mathbf{K}(\mathrm{mEq} / \mathrm{L})$ & $4.7 \pm 0.3$ & $4.6 \pm 0.4$ & $4.8 \pm 0.1$ & $4.6 \pm 0.4$ & 0.428 \\
\hline Urine microalbumin $^{+}(\mathrm{mg} / \mathrm{dl})$ & $114 \pm 314$ & $241 \pm 937$ & $40 \pm 78$ & $26 \pm 75$ & $P<0.001$ \\
\hline $\mathbf{W B C}\left(\times 10^{3} / \mu \mathrm{L}\right)$ & $8.1 \pm 1.5$ & $7.8 \pm 2.0$ & $7.8 \pm 1.9$ & $7.2 \pm 2.0$ & 0.401 \\
\hline $\mathbf{R B C}\left(\times 10^{6} / \mu \mathrm{L}\right)$ & $4.6 \pm 0.5$ & $4.6 \pm 0.7$ & $4.8 \pm 0.5$ & $5.2 \pm 0.6$ & 0.007 \\
\hline $\mathbf{H b}(\mathrm{g} / \mathrm{dL})$ & $13.0 \pm 1.4$ & $13.2 \pm 1.7$ & $13.6 \pm 1.8$ & $14.7 \pm 1.5$ & 0.001 \\
\hline Het (\%) & $39.5 \pm 4.6$ & $40.5 \pm 5.5$ & $41.5 \pm 5.1$ & $44.1 \pm 4.4$ & 0.005 \\
\hline PLT $\left(\times 10^{3} / \mu \mathrm{L}\right)$ & $254 \pm 54$ & $267 \pm 79$ & $259 \pm 53$ & $239 \pm 58$ & 0.483 \\
\hline MCV (fL) & $85.5 \pm 6.9$ & $85.8 \pm 7.1$ & $84.1 \pm 4.9$ & $86.0 \pm 5.4$ & 0.866 \\
\hline MCH (pg) & $28.2 \pm 2.3$ & $28.6 \pm 3.7$ & $28.3 \pm 2.9$ & $28.7 \pm 2.1$ & 0.904 \\
\hline $\mathbf{M C H C}^{+}(\mathrm{g} / \mathrm{dl})$ & $32.9 \pm 1.3$ & $32.8 \pm 2.4$ & $32.7 \pm 1.3$ & $33.4 \pm 1.2$ & ${ }^{+} 0.204$ \\
\hline $\mathbf{R D W}^{+}(\%)$ & $14.2 \pm 1.4$ & $14.6 \pm 1.7$ & $13.9 \pm 1.6$ & $13.9 \pm 1.3$ & ${ }^{+} 0.529$ \\
\hline \multicolumn{6}{|c|}{ *: One-way Anova test results, ${ }^{+}:$Kruskal Wallis test results; } \\
\hline \multicolumn{6}{|c|}{$P<0.05$ was considered statistically different and was indicated as bold. } \\
\hline \multicolumn{6}{|c|}{$\begin{array}{l}\text { PDR: Proliferative diabetic retinopathy, non-PDR: Non-proliferative diabetic retinopathy, AMD: Age-related macular degeneration, } \\
\text { BMI:Body mass index, AST: aspartate aminotransferase, ALT: alaline aminotransferase, CRP: C-reactive protein, BUN: Blood urine nitrogen, } \\
\text { Na: Sodium, K: Potassium, }\end{array}$} \\
\hline \multicolumn{6}{|c|}{$\begin{array}{l}\text { WBC: White blood cell, RBC: Red blood cell, Hb: Hemoglobin, Hct: Hematocrit, PLT: Platelet, MCV: Mean corpuscular volume, MCH: Mean } \\
\text { corpuscular hemoglobin, MCHC: Mean corpuscular hemoglobin concentration and RDW: Red cell distribution width. }\end{array}$} \\
\hline
\end{tabular}

DM follow-up blood parameters for $\mathrm{HbAlc}$ were $8.4 \mathrm{mg} / \mathrm{dL} \pm 1.9$ in group $1,8.1 \mathrm{mg} / \mathrm{dL} \pm 1.9$ in group 2 , $5.6 \mathrm{mg} / \mathrm{dL} \pm 0.5$ in group 3 , and $5.7 \mathrm{mg} / \mathrm{dL} \pm 0.5$ in group $4(P<0.001)$. Fasting glucose levels were $201 \mathrm{mg} / \mathrm{dL} \pm$ 87 in group $1,179 \mathrm{mg} / \mathrm{dL} \pm 71$ in group $2,106 \mathrm{mg} / \mathrm{dL} \pm 70$ in group 3 , and $98 \mathrm{mg} / \mathrm{dL} \pm 33 \mathrm{mg} / \mathrm{dL}$ in group $4(P<$ 0.001 ). Post hoc analysis showed statistically significant differences in HbA1c and fasting glucose levels among PDR-AMD, PDR-control, non-PDR-AMD, and non-PDR control (whole, $P<0.001$ ). Post hoc analyses of statistically significant parameters are shown in Table 2.

Serum and urine kidney parameters post hoc analysis showed statistically significant differences in creatinine levels between PDR-non-PDR and PDR-control $(P=0.034$ and $P=0.028$, respectively) and in urine microalbumin between PDR-AMD, PDR-control, non-PDR-AMD and non-PDR-control $(P<0.001, P=0.001$, $P=0.017$, and $P=0.048$, respectively). 
Table 2. Post hoc analysis of statistically significant parameters

\begin{tabular}{|c|c|c|c|c|c|c|}
\hline $\begin{array}{l}\text { Characteristics and } P^{*} \\
\text { values }\end{array}$ & PDR-nonPDR & PDR-AMD & PDR-control & nonPDR-AMD & nonPDR-control & AMD-control \\
\hline Hba1c (\%) & 1.000 & $P<0.001$ & $P<0.001$ & $P<0.001$ & $P<0.001$ & 1.000 \\
\hline Fasting Glucose (mg/dl) & 0.885 & $P<0.001$ & $P<0.001$ & $P<0.001$ & $P<0.001$ & 1.000 \\
\hline Creatinine (mg/dl) & 0.034 & 0.146 & 0.028 & 1.000 & 1.000 & 1.000 \\
\hline Albumin (mg/dl) & 1.000 & 0.005 & 0.047 & 0.058 & 0.157 & 1.000 \\
\hline $\begin{array}{l}\text { Urine } \quad \text { microalbumi } \\
(\mathrm{mg} / \mathrm{dl})\end{array}$ & + & $P<0.001$ & 0.001 & 0.017 & 0.048 & 1.000 \\
\hline $\mathbf{R B C}\left(\mathrm{x} 10^{6} / \mu \mathrm{L}\right)$ & 1.000 & 1.000 & 0.005 & 1.000 & 0.027 & 0.195 \\
\hline $\mathbf{H b}(\mathrm{g} / \mathrm{dL})$ & 1.000 & 0.737 & 0.001 & 1.000 & 0.005 & 0.098 \\
\hline Het (\%) & 1.000 & 0.533 & 0.003 & 1.000 & 0.045 & 0.406 \\
\hline \multicolumn{7}{|c|}{$\begin{array}{l}\text { *: post hoc analyze of Bonferroni test results, }{ }^{+}: \text {post hoc analyze of Dunn test results; } \\
P<0.05 \text { was considered statistically different and was indicated as bold. }\end{array}$} \\
\hline \multicolumn{7}{|c|}{$\begin{array}{l}\text { PDR: Proliferative diabetic retinopathy, non-PDR: Non-proliferative diabetic retinopathy, AMD: Age-related macular degeneration, ALT } \\
\text { alaline aminotransferase, BUN: Blood urine nitrogen, Na: Sodium, RBC: Red blood cell, Hb: Hemoglobin, Hct: Hematocrit, }\end{array}$} \\
\hline
\end{tabular}

Serum liver parameters post hoc analysis showed statistically significant differences in albumin between PDR-AMD and PDR-control groups ( $P=0.005$ and $P=0.047$, respectively).

No differences were found in CRP levels among all groups.

Post hoc analysis of $\mathrm{CBC}$ parameters showed statistically significant differences in $\mathrm{RBC}, \mathrm{Hb}$, and Htc values between PDR-control and non-PDR-control $(P=0.005, P=0.027 ; P=0.001, P=0.005 ; P=0.003, P=0.045$, respectively). Table 2 and Figure 1.

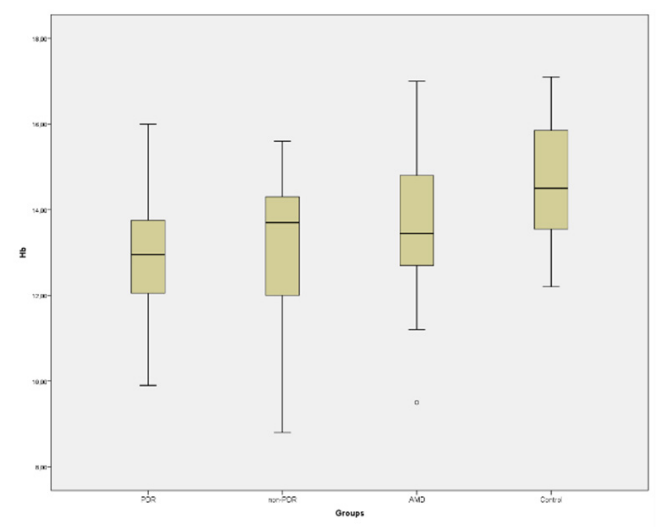

Figure 1. Haemoglobin levels in patients and controls. Hb, haemoglobin; PDR, proliferative diabetic retinopathy; non-PDR, nonproliferative diabetic retinopathy

Post hoc analysis of serum uric acid levels showed statistically significant differences in uric acid levels among PDR-non-PDR $(P=0.004)$, PDR-AMD $(P=0.019)$ and PDR-control $(P=0.004)$ groups respectively.

We found that mean $\mathrm{HbA1C}$ significantly correlated with triglycerides $(\mathrm{r}=0.252, \mathrm{p}=0.03)$. Triglycerides significantly correlated with urinary microalbumin $(\mathrm{r}=0.468, \mathrm{p}<0.001)$ and $\operatorname{Hct}(\mathrm{r}=-0.298, \mathrm{p}=0.015)$ levels in all groups ( $\mathrm{n}=138)$.

\section{Discussion}

In this study showed that there was a significant difference between biochemical parameters such as HbAlc, fastening glucose, creatinine, albumin, uric acid, urin parameters such as urinary microalbumin and haemogram parameters such as $\mathrm{Hb}, \mathrm{Hct}, \mathrm{RBC}$ in patients with PDR, non-PDR and advanced AMD.

There are studies showing that there is a correlation between the variability in clinical parameters such as Hba1c level, serum cholesterol and uric acid and the risk of complications of type 2 diabetes (Magri et al., 2012). $\mathrm{HbAlc}$ and fasting glucose tests are used in the diagnosis and follow-up of diabetes patients by Bennett et al., 
2007 and the American Diabetes Association in 2018 classification standards. In uncontrolled and advanced diabetic patients, these tests reveal elevations in these levels and necessitate treatment to regulate them (Barbieri et al., 2015). So patients diagnosed with DRP receive regular follow-ups to check their biochemical, haematological and urine parameters. Although this is not always the case in AMD patients, it is important to treat both these diseases systemically and perform regular follow-up lab parameter assessments.

Anaemia is often found in advanced diabetes patients. In our study, we found low RBC, Hb and Htc in both PDR and non-PDR groups. Recent evidence suggests a role for anemia in the development and progression of micro and macro vascular complications of diabetes (Sepulveda et al., 2012; Traveset et al., 2016; Bahar et al., 2013; Ajoy Mohan et al., 2011; Sonmez et al., 2010). Because Hb plays a substantial role in carrying oxygen throughout the body, decreased $\mathrm{Hb}$ may cause direct organ damage as a possible mechanism responsible for this association (Irace et al., 2011).

Insulin is an important regulator of albumin synthesis in addition to blood glucose. The relation between insulin deficiency and hypoalbuminemia may explain the high mortality rate in hyperglycaemic individuals with low serum albumin concentrations (González et al., 2013). In our study, we did perceive hypoalbuminemia in the PDR group, likely attributed to the fact that this group contained patients with uncontrolled diabetes.

Serum uric acid is the product of endogenous and exogenous purine metabolism and its derivatives. In the purine metabolic pathway, adenosine has vasoactive properties important to retinal blood flow. Adenosine produces superoxide nitric oxide, which affects retinal circulation by causing capillary obstruction, pericytes apoptosis and basement membrane thickening (Xia et al., 2014). These changes are seen in the pathogenesis of diabetic retinopathy. Previous cross-sectional studies of the relationship between serum uric acid level and DR have provided mixed results. While Vinuthinee-Naidu et al. showed that serum uric acid levels were not well-correlated to retinal nerve fibre layer and macular thickness among type 2 diabetic patients (Vinuthinee-Naidu et al., 2017) and older studies did not detect a relationship (Segato, 1991; Olukoga, 1991), other studies have shown that serum uric acid levels, diabetes and diabetic retinopathy are strongly correlated (Xia et al., 2014; Causevic et al., 2010). In our study, we found high uric acid levels in the PDR group.

Subramani et al. found a potential association between serum uric acid and neovascular AMD (Subramani et al., 2010). Uric acid, despite being a major non-enzymatic antioxidant in the human plasma, may function as pro-oxidant in certain situations, and seem to target predominantly lipids (Sautin et al., 2008). Čolak et al. (2017) found a positive correlation among uric acid and CRP levels and AMD.

In our study, however, no difference was found in CRP or uric acid levels in the AMD group. CRP is the major acute-phase reactant, and an active regulator of the innate immune system; has been one of the most widely studied putative plasma biomarkes for AMD with somewhat inconsistent results.

Similar to our results, two cross-sectional population-based study stated that there is no evidence that CRP levels are associated with AMD (McGwin et al., 2005; Klein et al., 2005). In a study with over 5000 aged British subjects, higher serum CRP levels were associated with increased risk of AMD in the longitudinal, but not in the cross-sectional analysis (Yip et al., 2015).

In terms of early diagnosis and treatment changes, a clinical examination, as well as biochemical, urine and haematological tests, are both appropriate and necessary.

\section{Conclusion}

This study showed that significant differences in biochemical, haematological, and urine values are present between AMD and DRP patients and healthy individuals. These differences may be due to the varying pathophysiologies of the DRP and AMD diseases, also indicated by different serum values between the two groups.

\section{Disclosure Statement}

All authors certify that they have no affiliations with or involvement in any organization or entity with any financial interest or non-financial interest in the subject matter or materials discussed in this manuscript.

\section{Ethics Approval and Consent to Participate}

The study adheres to the Tenets of the Declaration of Helsinki and the study is approved by the local ethical board the Clinical Research Ethics Committee.

\section{Conflict of interests}

The authors declare that there is no conflict of interests regarding the publication of this paper. 


\section{Abbreviations}

DM: Diabetes Mellitus.

AMD: Age-related Macular Degeneration.

DR: Diabetic Retinopathy.

PDR: Proliferative Diabetic Retinopathy.

non-PDR: Non- Proliferative Diabetic Retinopathy.

CRP: C Reactive Protein.

\section{References}

Ajoy Mohan, V. K., Nithyanandam, S., \& Idiculla, J. (2011). Microalbuminuria and low hemoglobin as risk factors for the occurrence and increasing severity of diabetic retinopathy. Indian Journal of Ophthalmology, 59(3), 207-210.

Amanita, M., \& Grzybowski, A. (2016). The role of the reactive oxygen species and oxidative stress in the pathomechanism of the age-related ocular diseases and other pathologies of the anterior and posterior eye segments in adults. Oxid Med Cell Longev, 3164734.

Ambati, J. F. B. J. (2012). Mechanisms of age-related macular degeneration. Neuron, 75(1), 26-39.

American Diabetes Association Classification and diagnosis of diabetes. (2016). Diabetes Care, 39, S13-S22.

American Diabetes Association Classification and diagnosis of diabetes: Standards of medical care in Diabetes. (2018). Diabetes Care, S13-27.

Asif, M. (2014). The prevention and control the type-2 diabetes by changing lifestyle and dietary pattern. $J E d u c$ Health Promot, 3(1).

Bahar, A., Kashi, Z., Amiri, A. A., \& Nabipour, M. (2013) Association between diabetic retinopathy and hemoglobin level. Caspian J Intern Med, 4, 759-762.

Barbieri et al. (2015). Anemia in Patients with Type 2 Diabetes Mellitus. Anemia Published Online First.

Bennett, C. M., Guo, M., \& Dharmage, S. C. (2007). HbA1c as a screening tool for detection of Type 2 diabetes: A systematic review. Diabet. Med., 24, 333-43.

Boekhoorn, S. S., Vingerling, J. R., \& Witterman, J. C. (2007). C-reactive protein level and risk of aging macula disorder. Arch Ophthalmology, 125(10), 1396-1401.

Causevic, A., Semiz, S., Dzankovic, A. M., Cico, B., ..., \& Bego, T. (2010). Relevance of uric Acid in progression of type 2 diabetes mellitus. Bosnian Journal of Basic Medical Sciences, 10(1), 54-59.

Čolak, E., Ignjatović, S., Radosavljević, A., \& Žorić, L. (2017). The association of enzymatic and non-enzymatic antioxidant defense parameters with inflammatory markers in patients with exudative form of age-related macular degeneration. Journal of Clinical Biochemistry and Nutrition, 60(2), 100-107.

Colijn et al. (2019). Increased high-density lipoprotein levels associated with age-related macular degeneration. Evidence from the EYE-RISK and European Eye Epidomiology Consortia. Opthalmology, 393-406.

Guariguata, L., Whiting, D. R., Hambleton, I., Beagley, J., Linnenkamp, U., \& Shaw, J. E. (2014). Global estimates of diabetes prevalence for 2013 and projections for 2035. Diabetes Res Clin Pract., 103(2), 137-149.

Infantino, C. A. G., González, C. D., Sánchez, R., \& Presner, N. (2013). Hyperglycemia and hypoalbuminemia as prognostic mortality factors in patients with enteral feeding. Nutrition, 29, 497-501.

Irace et al. (2011). Association among low whole blood viscosity, haematocrit, haemoglobin and diabetic retinopathy in subjects with type 2 diabetes. Br. J. Ophthalmol., 95, 94-98.

Klein et al. (2014). Harmonizing the classification of age-related macular degeneration in the three continent AMD consortium. Ophthalmic Epidemiol, 21, 14-23.

Klein, R., Klein, B. E., Knudtson, M. D., Wong, T. Y., Shankar, A., \& Tsai, M. Y. (2005). Systemic markers of inflammation endothelial dysfunction, and age-related maculopathy. Am J Ophthalmol., 140, 35-44.

Kunchithapautham, K., Atkinson, C., \& Rohrer, B. (2014). Smoke exposure causes endoplasmic reticulum stress and lipid accumulation in retinal pigment epithelium through oxidative stress and complement activation. $J$ Biol Chem., 289(21), 14534-14546. 
Liew, G., Joachim, N., Mitchell, P., Burlutsky, G., \& Wang, J. J. (2016). Validating the AREDS simplified severity scale of age related macular degeneration with 5- and 10-Year Incident Data in a Population-Based Sample. Ophthalmology, 123(9), 1874-8.

Magri, C. J., Calleja, N., Buhagiar, G., Fava, S., \& Vassallo, J. (2012). Ankle-brachial index in a type 2 diabetic population with proliferative retinopathy: Associated risk factors and complications. Int Angiol., 31(2), $134-41$.

Mauricio, D. (2016). Lower Hemoglobin Concentration Is Associated with Retinal Ischemia and the Severity of Diabetic Retinopathy in Type 2 Diabetes. Journal of Diabetes Research, 3674946.

McGwin, G., Hall, T. A., Xie, A., \& Owsley, C. (2005). The relation between C reactive protein and age related macular degeneration in the Cardiovascular Health Study. Br J Ophthalmol., 89(9), 1166-70.

Mitchell, P., Liew, G., Gopinath, B., \& Wong, T. Y. (2018). Age-related macular degeneration. Lancet. 29 , 392(10153), 1147-1159.

Olukoga, A. O., Erasmus, R. T., Akinlade, K. S., Okesina, A. B., Alanamu, A. A., \& Abu, E. A. (1991). Plasma urate in diabetes: Relationship to glycaemia, glucose disposal, microvascular complications and the variations following oral glucose. Diabetes Res Clin Pract Published Online First.

Paun et al. (2015). Genetic variants and systemic complement activation levels are associated with serum lipoprotein levels in age-related macular degeneration. Invest Ophthalmol Vis Sci., 56, 7766-7773.

Prakash, G., Agrawal, R., \& Natung, T. (2017). Role of lipids in retinal vascular and macular disorders. Indian J Clin Biochem., 32(1), 3-8.

Sautin, Y. Y., \& Johnson, R. J. (2008). Uric acid: The oxidant-antioxidant paradox. Nucleosides Nucleotides Nucleic Acids., 27(6), 608-619.

Segato, T., Midena, E., Grigoletto, F., Zucchetto, M., ..., \& Crepaldi, G. (1991). The epidemiology and prevalence of diabetic retinopathy in the Veneto Region of North East Italy. Diabet Med Published Online First.

Sepulveda, F. J., Perez, P., Medinilla, M. G., \& Aboytes, C. A. (2012). Anemia as a factor related to the progression of proliferative diabetic retinopathy after photocoagulation. J. Diabetes Complications, 26, 454-457.

Sivaprasad, S., Gupta, B., Crosby-Nwaobi, R., \& Evans, J. (2012). Prevalence of Diabetic Retinopathy in Various Ethnic Groups: A Worldwide Perspective. Surv. Ophthalmol., 57, 347-70.

Sonmez, A., Yilmaz, M. I., Saglam, M., Kilic, S., ..., \& Zoccali, C. (2010). The relationship between hemoglobin levels and endothelial functions in diabetes mellitus. Clinical Journal of the American Society of Nephrology: CJASN, 5(1), 45-50.

Stewart, M. W. (2010). Pathophysiology of diabetic retinopathy. Diabet Retin Evidence-Based Manag, 1-30.

Subramani, S., Khor, S. E., Livingstone, B. I., \& Kulkarni, U. V. (2010). Serum uric acid levels and its association with age-related macular degeneration (ARMD). Med J Malaysia, 65, 36-40. Retrieved from http://europepmc.org/abstract/MED/21265246

Traveset, A., Rubinat, E., Ortega, E., Alcubierre, N., ..., \& Mauricio, D. (2016). Lower Hemoglobin Concentration Is Associated with Retinal Ischemia and the Severity of Diabetic Retinopathy in Type 2 Diabetes. Journal of Diabetes Research, 3674946.

Vinuthinee-Naidu, M. N., Zunaina, E., Azreen-Redzal, A., \& Nyi-Nyi, N. (2017). Correlation of retinal nerve fibre layer and macular thickness with serum uric acid among type 2 diabetes mellitus. BMC Ophthalmol, 17.

Xia, J., Wang, Z., \& Zhang, F. (2014). Association between related purine metabolites and diabetic retinopathy in type 2 diabetic patients. Int $J$ Endocrinol.

Yip et al. (2015). Cross sectional and longitudinal associations between cardiovascular risk factors and age related macular degeneration in the EPIC-Norfolk eye study. PLoS One, 10, e0132565.

\section{Copyrights}

Copyright for this article is retained by the author(s), with first publication rights granted to the journal.

This is an open-access article distributed under the terms and conditions of the Creative Commons Attribution license (http://creativecommons.org/licenses/by/4.0/). 